Teilnehmen kann jeder, der bis zum 15.08.2012 eine Idee für die Nutzung der Online-Funktion des Personalausweises hat und seine eIDEE unter MITMACHEN auf der Website $h t t p: / / w w w . d i g i t a-$ ler-handschlag.de/ kurz beschreibt.

\section{Intel und TU Darmstadt eröffnen Forschungsinstitut für Secure Computing}

Der Halbleiterhersteller Intel eröffnet in Darmstadt gemeinsam mit der TU das Intel Collaborative Research Institute for Secure Computing (ICRI-SC) - das erste Intel-Forschungszentrum für IT-Sicherheit außerhalb der USA, das seinen Sitz am Center for Advanced Security Research Darmstadt (CASED) haben wird. Besonders im Fokus: Die Sicherheit von mobilen Geräten. Die Wissenschaftler suchen neue Wege, um die Zuverlässigkeit und Sicherheit von mobilen Geräten zu steigern.

Geleitet wird das Institut von Institutsdirektor Prof. Ahmad-Reza Sadeghi vom Lehrstuhl System Security an der TU Darmstadt und dem angehenden Chief Technologist des Instituts, Dr. Matthias Schunter von Intel.

„Moderne IT-Systeme müssen mit größtmöglicher Sicherheit ausgestattet sein. Dies ist umso wichtiger, da moderne Informations- und Kommunikationstechnologien als Querschnittstechnologien in alle Wirtschaftsbereiche vordringen, und damit Motor für wirtschaftliche und gesellschaftliche Entwicklungen sind", sagte der hessischen Staatssekretär für Wirtschaft, Verkehr und Landesentwicklung Steffen Saebisch. „Dieser Transfer in Produkte und Verfahren ist uns sehr wichtig. Deshalb unterstützt das Hessische Wirtschaftsministerium die Transferaktivitäten des Institutes mit EU-Strukturfondsmitteln in Höhe von insgesamt 180000 Euro über die nächsten drei Jahre".

Das Institut ist bereits Teil der heute bekanntgegebenen neuen Forschungsstrategie von Intel: ein weltweites Netzwerk akademischer Forschungskooperationen, den sogenannten Intel Collaborative Research Institutes (ICRI). Das ICRI-Programm basiert auf den in den USA sehr erfolgreichen Intel Science and Technology Centers (ISTCS), die Wissenschaft und Wirtschaft zusammenbringen, um eine neue Generation von Technologien vorherzusehen und zu verwirklichen. „Ziel ist es, globale Innovation in wichtigen Bereichen voranzutreiben und dazu beizutragen, sich einigen der heute größten Herausforderungen zu stellen", sagte Intel CTO Justin Rattner.

„Zukünftige IT-Technologie wird so aussehen, dass viele verborgene Prozessoren über ein verteiltes Betriebssystem, eine Art „Über-Cloud" den Anwendern die Nutzung verschiedener Dienste und Geschäftsmodelle erlauben: von der ,Cloud of Devices' bis hin zur car-to-x communication", sagt Institutsdirektor Ahmad-Reza Sadeghi. „In Darmstadt suchen wir zusammen mit Wissenschaftlern von Intel neue Wege, um die Zuverlässigkeit und Sicherheit von zukünftigen mobilen Geräten und eingebetteten Systemen sowie von dem angeschlossenen Ökosystem maßgeblich zu verbessern."

\section{Eingebettete Systeme für Smart Devices}

Dafür entwerfen die Forscher Sicherheitsarchitekturen und Mechanismen für die Integritätsüberprüfung von eingebetteten Systemen und insbesondere für Smart Devices, z.B. Smartphones und Tablets. Zum Beispiel suchen die Wissenschaftler nach neuen Ansätzen für Mobile Commerce und versuchen ein besseres Verständ- nis für Privatsphäre und deren Realisierung zu entwickeln. Zudem werden dort Lösungen zur fehlerfreien Kommunikation zwischen Fahrzeugen und eingebetteten Geräten entwickelt, die eine erhöhte Sicherheit beim Autofahren garantieren. Basierend auf der Erforschung der Bedürfnisse künftiger Nutzer, wird das Institut passende Soft- und Hardware entwickeln, die robuste und zuverlässige Systeme für diese Anwendungsszenarien bereitstellen.

\section{E-Postident - Instrument für den Jugendschutz im Internet}

Der neue Online-Identifikationsservice E-Postident hat von der Kommission für Jugendmedienschutz der Landesmedienanstalten (KJM) die Positivbewertung als „übergreifendes Jugendschutz-Konzept“ erhalten. Online-Händler können mit E-Postident nun vollautomatisiert und in Echtzeit sicherstellen, dass altersbeschränkte Content- und Software-Downloads ausschließlich von volljährigen Kunden erworben werden.

Der Online-Altersnachweis via E-Postident funktioniert schnell und einfach. Internetnutzer, die sich für den E-Postbrief registriert haben, klicken beim Kauf altersbeschränkter Inhalte einfach auf den Button „E-Postident" auf der Website des Anbieters. Sie werden automatisch auf den sicheren E-Postbrief Server der Deutschen Post umgeleitet. Dort geben sie ihre E-Postbrief Adresse, ihr Passwort sowie die TAN ein, die sie auf ihr Handy geschickt bekommen. Das E-Post System bestätigt dann dem Download-Anbieter die Volljährigkeit des Kunden - fertig. „E-Postident ist einfach und kostensparend für Händler, bequem für die Verbraucher - und dabei für alle Beteiligten vertraulich und rechtssicher", sagt Ralph Wiegand, CEO E-Postbrief der Deutschen Post.

Die Einsatzmöglichkeiten von E-Postident gehen aber über die reine Altersverifikation hinaus: Je nach Bedarf können Online-Anbieter Namen, Geburtsdaten oder Adresse ihrer Kunden zweifelsfrei bestätigen lassen. Übermittelt werden an den Website-Betreiber nur die Angaben, deren Weitergabe der Nutzer ausdrücklich zugestimmt hat. Er hat jederzeit die volle Kontrolle über seine persönlichen Daten. Aufgrund der steigenden Betrugsraten im Internet kommt die Deutsche Post somit dem Bedarf nach geprüften Identitäten nach.

Mit E-Postident erhält bereits das zweite Online-Angebot der Deutschen Post das KJM-Siegel. Bereits im September 2011 erteilte die Kommission für Jugendmedienschutz dem E-Postbrief ihr Gütesiegel.

\section{Neuer Standard für die Java-Programmierung von TPM}

Private PCs und komplette Firmennetzwerke sind heute schwerwiegenden Bedrohungen ausgesetzt. Malware, Trojaner, Viren und andere virtuelle Quälgeister finden mit Garantie eine Schwachstelle im System, dringen ein und richten mitunter immense Schäden an. Dagegen wendet sich das „Trusted Platform Module (TPM)“. Dieser Sicherheitschip, der bereits von nahezu allen namhaften PCund Notebook-Herstellern in ihre höherklassigen Rechner integriert wird, soll die Unversehrtheit der Software sicherstellen. Er prüft, ob auf einem PC nur die gewünschte Software läuft. Eine 